\title{
Convergence of ICN and MEC for 5G: Opportunities and Challenges
}

\author{
Gürkan Gür, Senior Member, IEEE, Pawani Porambage, Member, IEEE, \\ Madhusanka Liyanage, Senior Member, IEEE
}

\begin{abstract}
Information-Centric Networking (ICN) builds on a content-centric network architecture to overcome the shortcomings of host-centric routing/operation and realize efficient pervasive and ubiquitous networking. Similarly, Multi-access Edge Computing (MEC) is another key technology to fulfill the stringent requirements of $5 \mathrm{G}$ to offer anytime-anywhere connected services for massive number of devices with ultralow delay and very large bandwidths. The amalgamation of ICN paradigm with MEC opens up new opportunities as well some challenges to realize $5 \mathrm{G}$ vision and advance beyond 5G systems. The key motivation of this paper is to discuss and elaborate the convergence of ICN and MEC for better future networks, and to identify the current standardization efforts. Moreover, the paper renders the key use cases and identifies potential research directions with the coexistence of ICN and MEC.
\end{abstract}

Index Terms-Multi-Access Edge Computing (MEC), Information-Centric Networking (ICN), network softwarization, Internet of Things (IoT), 5G.

\section{INTRODUCTION}

The high-bandwidth and ultra-low latency services in $5 \mathrm{G}$ networks accompanied with the critical cost efficiency requirement have led to pivotal ideas on optimizing spatial configuration of services in these systems. This rationale is embodied in the Multi-access Edge Computing (MEC) concept which is based on the basic premise of placing generic compute and storage resources with advanced applications close to the mobile network edge. Another important proposal for future networks is to change the overall underlying networking paradigm to cope with the shortcomings of current Internet infrastructure. In that regard, Information-Centric Networking $(I C N)$ is an Internet architecture that is built around the information concept and replaces the client-server model by proposing a new publish-subscribe model [1].

The key benefits of MEC stem from the fundamental paradigm of providing services in a spatially-optimized setting. They include proximity, ultra-low latency, high bandwidth, location awareness and contextualized services. From the service deployment and provisioning perspective, MEC enables

Gürkan Gür is with Zurich University of Applied Sciences (ZHAW), Switzerland. e-mail:gueu@zhaw.ch

Pawani Porambage is with the Center for Wireless Communications, University of Oulu, Finland. e-mail:pawani.porambage@oulu.fi

Madhusanka Liyanage is with the School of Computer Science, University College Dublin, Ireland and the Center for Wireless Communications, University of Oulu, Finland. e-mail:madhusanka@ucd.ie

This research is funded by the Scientific and Technical Research Council of Turkey (TUBITAK) under Grant 116E245, European Union under RESPONSE 5G (Grant No: 789658) and the Academy of Finland under 6Genesis Flagship (grant 318927) projects. some applications (e.g. Augmented Reality/Virtual Reality $(\mathrm{AR} / \mathrm{VR})$ ) which are not practically feasible in service architectures centralized in the network core. It is also related to mobile network's operational challenges regarding bandwidth bottlenecks and provisioning. It allows consumers to use more connected applications and devices without congesting the core network since short service loops are possible without contaminating backhaul and core networks.

ICN is instrumental to support the ever burgeoning bandwidth hungry and low latency Internet applications such as $4 \mathrm{~K} / 8 \mathrm{~K}$ videos, 3D games, and AR/VR applications. It provides many benefits including high speed data delivery and high reliability for Future Internet [1]. This relatively novel technology focuses on utilizing caching, replication and content distribution in optimal ways [2]. The basic idea of ICN is to enrich network-layer functions with content awareness. Therefore, it is possible to do routing, forwarding, caching and data-transfer operations on topology-independent content names rather than on IP addresses [3]. In ICN, the contentretrieval process employs names that are assigned through different naming schemes such as hierarchical, flat, attribute, and hybrid (name-based routing) [4]. Additionally, a single content can be divided into multiple small segments called chunks in order to adapt the transport layer.

The name-based forwarding and routing mechanism in an ICN network builds on three fundamental data structures that capture a network node's state: a Forwarding Interest Table (FIB) (as seen in Fig. 1), a Pending Interest Table (PIT), and a Content Store (CS) [2]. CS is dedicated to the cache memory whereas PIT and FIB are essentially for forwarding and routing procedure. ICN also utilizes interest forwarding strategies which takes input from both FIB and measurements to make interest forwarding decisions. When a node receives an ICN interest packet, it locally checks its CS and PIT to find a matching name; if no match is found, it forwards the interest to the next hop(s) towards the requested content based on the information in its FIB after it records that interest in its PIT. Although there exist alternative approaches which aim at reducing the amount of state that a nodes must keep, up to fully PIT-less designs using packets for keeping state, the overall service model is fundamentally the same.

According to [5], a 5G network may use ICN for content distribution and transparent mobility among multiple access technologies, and also leverage ICN's ability to cope with link impairments in wireless networks. As an implementation option, an end-to-end ICN framework could be implemented as an overlay on existing IP networks (ICN over IP). ICN 
could also be deployed within a 5G network slice where separate ICN frames would be treated differently compared to IP packets. In essence, the integration of ICN into MEC environment is vastly beneficial for efficient realization of $5 \mathrm{G}$ and Beyond $5 \mathrm{G}$ promises, which motivates us to investigate the prospects of such systems in this article. To this end, the contributions of this work are as follows:

- We discuss key concepts and present the mutual benefits of ICN MEC integration via potential use-cases. (Section II and III)

- We elaborate on how ICN and MEC can be integrated in $5 \mathrm{G}$ networks with a review of challenges and solutions. (Section IV)

- To render the standardization aspect, we present current efforts of SDOs (Standards Developing Organizations) and describe their contributions. (Section V)

- Albeit its merits, this integration also has open challenges for Beyond $5 \mathrm{G}$ networks, which we discuss finally in the context of future research directions. (Section VI)

\section{Mutual Benefits of ICN AND MEC Integration}

Several benefits for $5 \mathrm{G}$ networks can be achieved by exploiting the synergy between ICN and MEC as depicted in Fig. 1. Moreover, Table I discusses the key benefits of ICN and MEC integration in $5 \mathrm{G}$ networks, which we discuss in this section.

1) Faster content delivery: MEC has designed to deliver content for delay critical applications. However, some of this content has to be fetched from cloud to the edge nodes. The use of ICN can enable high speed content migration between the MEC edge nodes and central cloud systems due to its advanced caching and content replication functions.

2) Improved application level reconfiguration: MEC based service applications may request the addition and/or removal of softcore components (i.e. VNFs) at any point in time. Such application level reconfiguration is challenging in MEC systems, since a session re-initialization is required whenever a session is being served by a non-optimal service instance. This process always increases the session migration delay and significantly impairs the low latency applications. Particularly, the mobility of MEC users will lead to network level changes in that regard.

Due to the service-centric networking characteristics in ICN technology, it allows fast resolution of named service instances [3]. Thus, ICN can improve application level reconfiguration by minimizing the network configuration delay for MEC applications.

3) Improved storage and caching functions at the edges: The coexistence of ICN and MEC can also improve the performance of storage and caching functions in edge networks. This is enabled by two key features of ICN: location independent naming, and data replication and opportunistic caching at strategic points in the network. These features benefit both realtime and non-realtime $5 \mathrm{G}$ applications where a set of users shares the same content [3].
4) Improved efficiency of session mobility: ICN introduces ubiquitous in-network caching to reduce network load and improve Quality of Service (QoS) of services. This feature is instrumental to reduce bandwidth utilization for signaling traffic for session mobility management in MEC system. Thus, the use of ICN will significantly improve the efficiency of session mobility in MEC networks and also reduce the operational cost.

In contrast to the anchor-based mobility approach used in current MEC networks, ICN uses a split between the application identifier and the name resolution for mobility management. By using these application bound identifier and location split principles, ICN can significantly reduce control and user plane overheads of session mobility events in MEC network and handles host mobility efficiently.

5) High mobility support for URLLC 5G applications: MEC systems are also advantageous in exploiting contextaware data distribution. The real time context aware applications could be accomplished with the correct coordination between MEC platforms. ICN provides considerable opportunities for context-aware data distribution in the networks by allowing content distribution over unreliable radio links and transparent mobility between heterogeneous network [6]. Due the latency support of MEC, ICN MEC integration is important to provide services for high mobility $5 \mathrm{G}$ applications such as tactile Internet and autonomous vehicles [7].

6) Better support for real-time context aware 5G applications: Real time context aware applications such as autonomous vehicles can be further facilitated via ICN MEC integration platforms by providing opportunistic caching at ICN access points. This will reduce unicast downlink transmissions at the service level and allow contextualized traffic for different user requirements. Basically, contextualized traffic stands for the predicted traffic which can be formulated by analysing the behaviours of the surrounding user requests and historical traffic profiles. Here, the likelihood of using an identical service by a group of users in close proximity can be evaluated leading to mitigation of redundant downlink unicast transmissions, which would otherwise increase the content delivery cost and network level resource (backhaul network resources) consumption for such applications.

Proper re-programming of the ICN layer in the ICN MEC integration may allow UEs to move from one Radio Access Network (RAN) instance to another and ease transparent mobility between heterogeneous networks [6]. It can be used to tackle link quality fluctuations, which is challenging in the physical and medium-access layer for real-time applications.

7) Content-based security: In ICN MEC integrated platforms, the content-based security offered by ICN will allow authentication during the content consumption. This will eliminate the additional authentication delays. Moreover, the self-certifying (or certificate-less) public key cryptography based naming models of ICN will help to increase security proprieties without relying on a third party.

8) Enabler for practical ICN deployments in the edge: MEC provides a flexible service environment in the edge networks. Such capabilities can facilitate gradual implemen- 

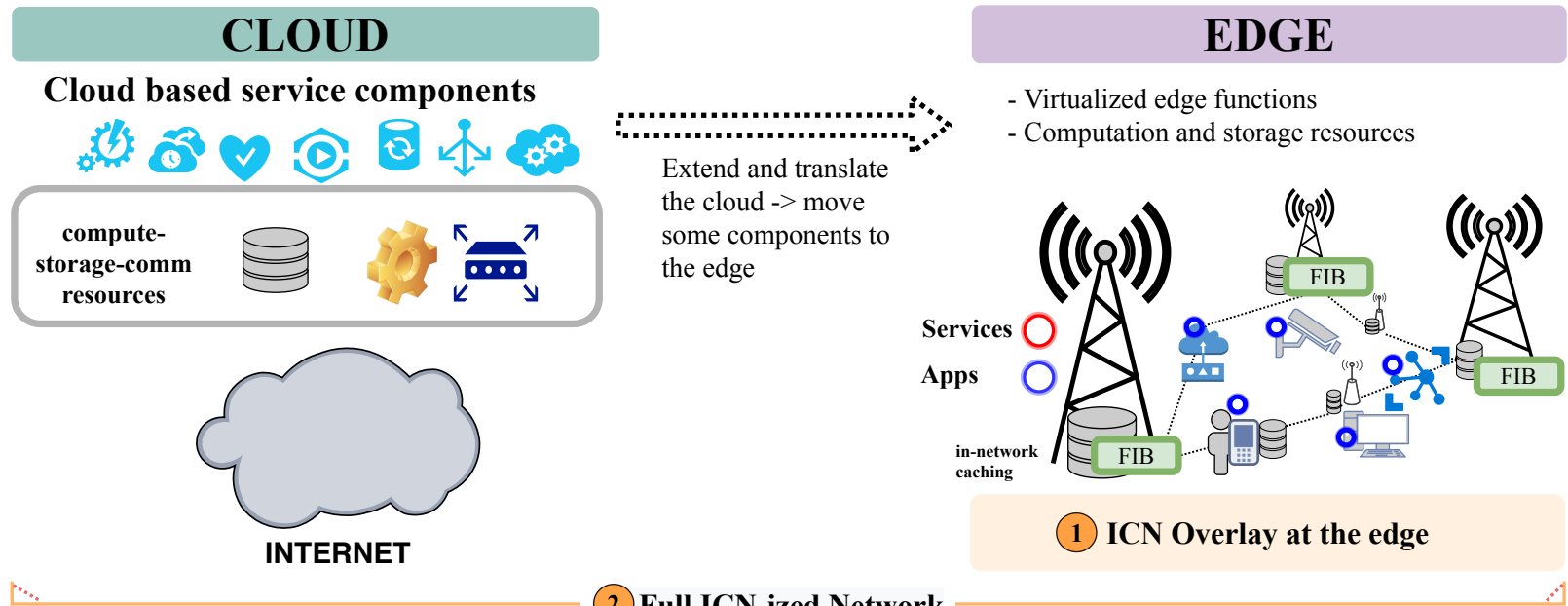

(2)Full ICN-ized Network

Fig. 1: Integration of ICN and MEC in 5G. ICN is a beneficial enabler for the edge computing scenarios (1) while it can be a much more fundamental networking technology in the long run (2).

TABLE I: Mutual benefits of ICN and MEC integration in 5G networks.

\begin{tabular}{|c|c|c|}
\hline Technology & Feature & Mutual Benefit \\
\hline \multirow{5}{*}{$\mathrm{ICN}$} & Service-centric networking & Reduces session migration delay in MEC to support the low latency applications \\
\hline & Function naming & $\begin{array}{l}\text { MEC can use ICN to allow devices to express the required services without specifying the exact } \\
\text { node. ICN can support to route the requests to the best matching MEC server and migrate network } \\
\text { functions. }\end{array}$ \\
\hline & Location independent naming & $\begin{array}{l}\text { Supports single abstraction of available data and computing resources so that they can access in } \\
\text { one universal way }\end{array}$ \\
\hline & Ubiquitous caching and replication & $\begin{array}{l}\text { Improves the consumer mobility. ICN caching provides a copy of the content to all users and no } \\
\text { need to send requests to the original server. }\end{array}$ \\
\hline & Opportunistic caching & $\begin{array}{l}\text { Opportunistic caching is beneficial spatial and temporal correlation among content accessed by the } \\
\text { users. This is useful for high-bandwidth and low-latency MEC based applications such as AR/VR, } \\
\text { and autonomous vehicle driving. }\end{array}$ \\
\hline \multirow{2}{*}{ MEC } & Storage at close proximity to user & $\begin{array}{l}\text { Enables ICN caching services at the MEC servers to improve user mobility and latency requirements } \\
\text { by offering more storage closer to the users for opportunistic caching }\end{array}$ \\
\hline & Service environment in the edge & Facilitates practical implementation of ICN segments for different use-cases \\
\hline
\end{tabular}

tation of ICN segments for different use-cases realizing the benefits of ICN paradigm. This practical aspect is particularly important to exploit benefits of ICN and thus promote its integration in the overall $5 \mathrm{G}$ environment and future wireless networks.

\section{USE CASES OF ICN MEC}

To lay out how ICN and MEC can be integrated in 5G, we describe three major $5 \mathrm{G}$ use cases in this section by covering all three 5G traffic classes, i.e. mMTC (massive Machine Type Communications), eMBB (enhanced Mobile Broadband), and URLLC (Ultra Reliable Low Latency Communications). Since this is a limited set of use cases for the sake of brevity, please note that it is not exhaustive (i.e., there are other use cases and applications to benefit from this integration.)

\section{A. IoT}

The well-known paradigm of IoT extends the capabilities of electronic devices and network connectivity tremendously. IoT represents a good example of the 5G traffic class mMTC, which refers to periodically-active IoT devices that send chunks of small data payloads. MEC will bring the cloud computing capabilities closer to the end user IoT devices [8]. ICN may introduce more information-oriented characteristics into IoT in such a way to match with the MEC scenarios.

IoT is intrinsically compliant with name-based protocols such as ICN since data sources such as sensors can translate to ICN URIs. For instance, let's assume a smart home (SH) scenario where energy consumption of gadgets at home are monitored by an SH service provider to minimize energy consumption and costs. In that case, we can name each device 


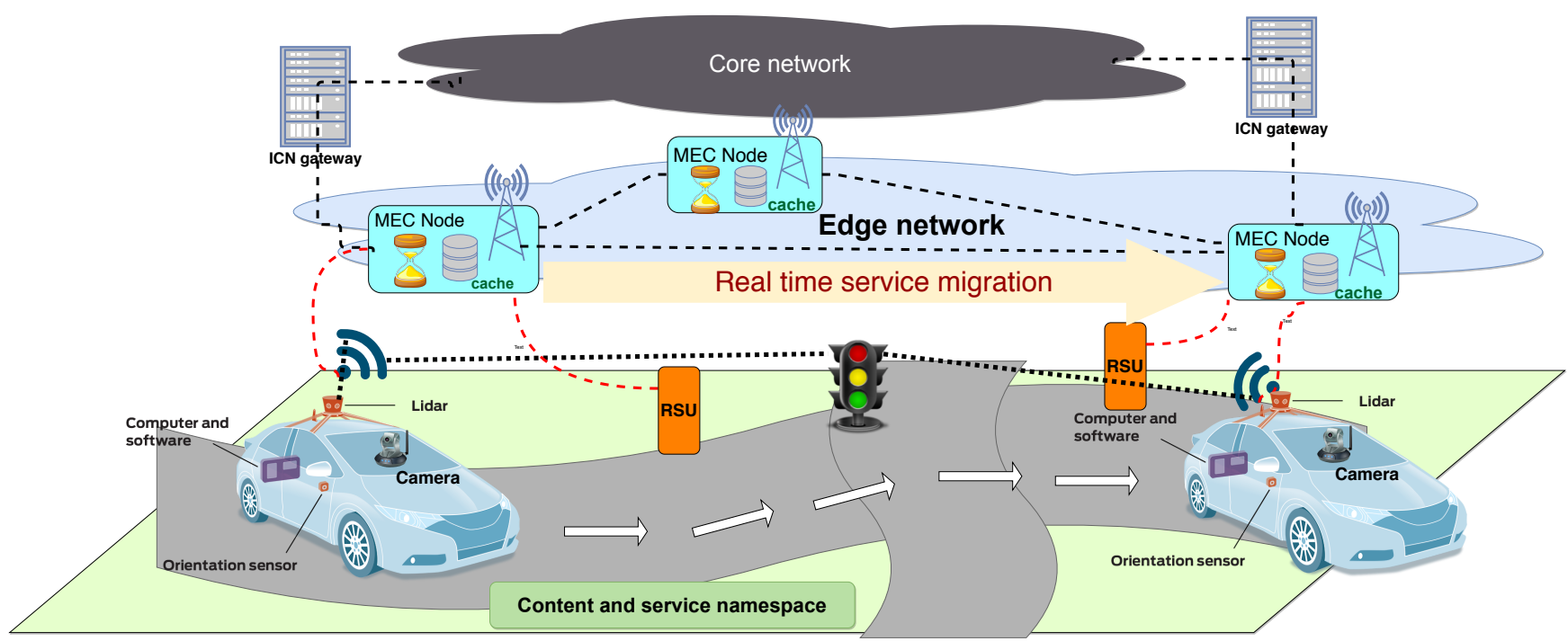

Fig. 2: Different use case realizations in information-centric MEC.

at home as /homeId/entity/task/location/ where the entity component specifies the end device (e.g. fridge), the task component describes which specific sensing or action task must be performed (e.g., meter reading, checking on/off state) and the location component identifies the physical position of the end device in the house. The central application sends an Interest (request packet) to ask for a measurement or an action, and waits for a Data packet from the end device containing the requested parameter value or the result of the triggered action. Moreover, in-network caching which refers to caching in network devices including MEC nodes can mitigate the amount of duplicate data floating in the network. Basically, it can decrease of transmissions of data (data-in-transmit) since it will be available in proximity with faster and retrieval via caching in ICN MEC, assuming welldesigned and implemented caching frameworks in the MEC environment. Caching can also increase the data availability to improve the reliability of IoT services. In the SH scenario, caching in different network elements in the edge network may serve some sensing data to other service providers which want to use the same data for other services (e.g. activity tracking for security). If implemented properly, the object security model in ICN provides embedded security in IoT environment. This capability is beneficial for network layer security and authentication of user requests to allow location-independent caching and computing. Overall, ICN MEC together will serve IoT in terms of name resolution, data collection and analytics, caching, service virtualization, and multicasting.

\section{B. Networked Multimedia Applications}

Efficient provision of these services with stringent QoS (Quality of Service) is crucial for 5G. ICN in MEC environment is instrumental for that goal. It allows for native video acceleration and delivery optimization for $4 \mathrm{~K} / 8 \mathrm{~K}$ video. It enables traffic optimization in the edge in addition to backhaul and core networks via in-network caching. Since cache management based on content popularity, request pattern, and quality awareness (user device type, available bandwidth, etc.) is possible, ICN provides traffic localization in the edge. Furthermore, it results in distributed, yet simpler streaming infrastructure. With multimedia applications and prevalent content caching in MEC environment, it is possible to have multiple active links and load balancing based on dynamic conditions. AR/VR services especially benefit from ICN due to native capabilities of seamless mobility, multi-path support and in-network caching. Heavy AR/VR processing loads can be distributed among edge nodes in the proximity of service consumers in ICN MEC. Specifically, information-centric edge network architecture provides better service optimization by monitoring the "small-scale" at the edge. Typically, network optimization schemes try to have a global view and optimize over a large scope. However, optimization inside the edge is beneficial for accelerated multimedia services. In that regard, ICN provides an additional layer of optimization to the spatial optimization of MEC paradigm.

\section{Autonomous Driving Cars}

The fully and semi-autonomous driving cars are important technologies to be supported by $5 \mathrm{G}$ networks. They are equipped with advanced sensory units and frequently communicate with the road-side units to obtain a detailed preview of the road ahead. These advanced sensory units range from cameras, GPS, radar, sonar to LIDAR (Light Detection and Ranging). This communication allows the car to obtain new features and functions such as adaptive cruise, predictive power-train control and adaptive navigation. However, a future car with full autonomy is expected to generate and utilize up to 4 TB data per day due to hundreds of on-vehicle sensors and advanced services (For instance, cameras alone will generate 20 to $40 \mathrm{Mbps}$ ) [9]. Moreover, lots of riders are now interested in accessing cloud based services such as HD video streaming and use various latency-sensitive mobile applications such as real-time online gaming and virtual sports. 


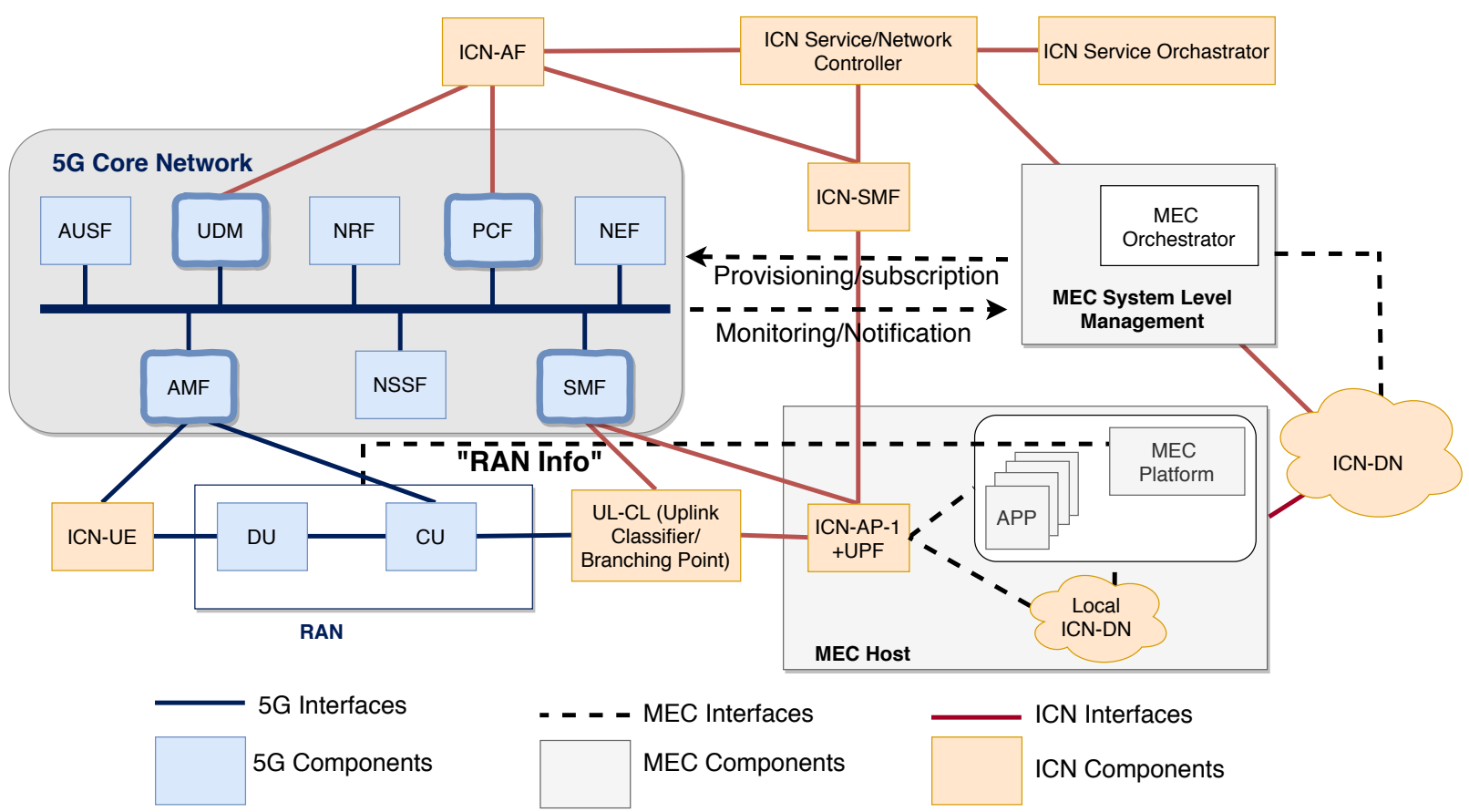

5G Core Network Functions

- UDM: Unified Data Management

- PCF: Policy Control function

- AMF: Access and Mobility Management Function

- SMF: Session Management Function
ICN Components

- ICN-SMF: ICN Session Management Function

- ICN-AF: ICN Application Function

- ICN-AP: ICN Anchor Point

- ICN-DN: ICN Data Network

- UPF: User Plane Functions

Fig. 3: A depiction of how ICN and MEC can be architecturally integrated.

Most of these connected applications require tactile speed with latency approaching $1 \mathrm{~ms}$. An edge-driven service environment with ICN and MEC for autonomous driving car usecase is depicted in Fig. 2. In this context, ICN can enhance the capability of MEC. For instance, ICN based producer mobility schemes can be used in ICN MEC systems to support high speed mobility events. Both anchor based and anchorless approaches can be utilized [10]. This will enable the high speed migration of control functions as well as other services to the MEC end hosts along the path of the car. This scenario can be further extended to enable the proactive content and result placement. With the help of ICN, it is possible to pre-fetch the popular common data such as local maps or weather information and store it within ICN caches to reduce the end-to-end latency. Many ICN based predictive and proactive caching/fetching strategies are promising to use in MEC systems to get computation results such as locationbased AR/VR data or situational awareness data, e.g. overthe-horizon proximity data for cars, or improve in-vehicle infotainment services [9].

\section{INTEGRATION CHALLENGES OF ICN AND MEC}

This section focuses on integration challenges that need to be addressed while designing an integrated ICN MEC architecture for $5 \mathrm{G}$.

\section{A. Deployment and Orchestration}

As per today, most of the ICN and MEC systems are deployed independently despite being complementary concepts [8]. To achieve their full benefit in 5G networks, joint deployment of these technologies should be explored. Several works had proposed 5G-MEC integration [11] and 5G-ICN integration [12] approaches separately. In integrated ICN MEC architecture, proper APIs (Application Programming Interfaces) have to be defined to enable the communication between MEC and ICN components in 5G networks. It is also important to develop efficient system control orchestrator and/or coordination architecture to enable their cooperation. Moreover, such architecture should focus on automatic and autonomic system control rather than the traditional provisioning/configuration or control of networking systems. The main drivers for such autonomic management include the complexity of ICN MEC architecture in addition to 5G itself with very stringent performance requirements, cost concerns for complicated network management operations, the anticipated QoS assurance for $5 \mathrm{G}$ use-cases and envisaged benefits provided by optimizations by ML/AI based control schemes acting for zero-touch and cognitive network management.

Possible Solutions: A possible integrated ICN MEC architecture for $5 \mathrm{G}$ is presented in Fig. 3 based on [12]. To realize the MEC ICN integration in 5G Core (5GC) network, four key Network Functions - NFs (i.e., Session Management 
Function - SMF, Unified Data Management - UDM, Policy Control Function - PCF and Access and Mobility Management Function - AMF) have to interface with ICN components.

SMF is primarily responsible for interacting with the decoupled data plane, managing Protocol Data Unit (PDU) sessions and session context. Similarly, ICN-SMF serves as control entity for the ICN state managed in ICN-APs (ICNAnchor Points). It is also responsible for management of dynamic name based forwarding states, mobility states, innetwork service functions, resources such as caching, storage, and compute resources for PDU session via ICN-APs. In addition, PDU sessions termination and ICN service network initiation are happening at ICN-APs. Therefore, the direct communication between ICN-APs and 5GC SMF is needed to manage the ICN sessions of UEs.

In 5GC, UPF acts as external PDU session point of interconnect to Data Network (DN) and supports packet routing and forwarding, packet inspection, QoS handling. Also, PCF provides policy rules to control plane functions for governing network behavior. The new ICN-AF interfaces with PCF and UDM to transfer user profile, subscription and authentication policies and details required to validate ICN-UE's PDU session requests. Those requests will be received via ICN-SMF interface. In addition, ICN-AF interfaces with ICN service and network controller to steer ICN PDU session traffic and serve edge service functions by updating policy rules of $5 \mathrm{G}$ networks.

In ICN enabled network, UEs must be authorized to access ICN DNs. AMF performs task such as the registration, access authentication and authorization, security context management for UEs. Therefore, ICN UEs have to communicate with AMF to get authorized for 5G services. ICN Service controller and MEC Orchestrator should have direct communication link to exchange the critical control information.

To support low latency $5 \mathrm{G}$ applications, some of the ICN components such as ICN-APs, Local ICN-DN should be implemented in MEC hosts. These entities will directly communicate with MEC edge apps to realize edge level communication. ICN-DN should be deployed in between core and edge networks. It minimizes the network configuration delay of MEC applications and offers fast resolution of named service instances.

\section{B. Context Awareness}

The real advantages of MEC can be achieved by obtaining context information such as user location, other users in vicinity, overall condition and resources in the environment. Although ICN can provide different levels of context information (application, network and device level), aggregation of these data is still challenging. In addition, most of the current ICN research is still focused on providing the basic functionality, rather than on utilizing the available context information to improve network parameters such as QoS and Quality of Experience (QoE). Therefore, ICN research has to focus on providing as much contextual information as possible for MEC systems.
Possible Solutions: ICN MEC integration can improve the context awareness by enabling new MEC based services such as Real-Time Location Systems (RTLS), Anonymous Video Analytics (AVA) and AR. RTLS is wireless system that automatically tracks the location of people or objects within a defined area. AVA can be used to gather information about a nearby person through camera sensors to detect patterns and identify details such as gender and age. It does not record images and, unlike facial recognition, does not match images against a database.

\section{Security and Privacy}

Key security vulnerabilities in ICN are identified as denial of service (DoS), cache pollution, and content poisoning attacks, whereas privacy issues are discussed in terms of user privacy and anonymity, name and signature privacy, and content privacy. As a result, additional security features have to be implemented in MEC systems.

Moreover, ICN systems can not use traditional access control schemes based on Access Control Lists (ACLs). The innetwork caching function of ICN enables the possibility to delivering Named Data Objects (NDOs) on-demand basis. In this environment, ICN entities have to maintain an identical control policy over NDOs for each consumer to support ACL based access control. However, such mechanism is challenging due to privacy issues and computational overhead.

Possible Solutions: The well-known access control schemes (e.g., encryption-based, attribute-based, session-based, and proxy re-encryption-based) can be also incorporated with ICN MEC integration for end-to-end security. In addition, MEC driven ICN traffic isolated as a separate network slice with better security provisioning is another approach. However, this is not a trivial solution since ICN MEC integrated systems face various challenges due to authorization and access control challenges in ICN systems. Simply, the content has to be secure with proper confidentiality and credentials while being uniquely identifiable among a huge number of objects present in the network. In that regard, user level authorization is a significant challenge in ICN due to lack of user-to-server authentication.

\section{Naming}

Naming related challenges are linked to the general challenges of ICN. First, which namespace structure is efficient in which context is an important research question, e.g., hierarchically structured versus flat names. Moreover, the namespace should be scalable and manageable in term of size. This is a key research topic regarding the massive connectivity and IoT. In general, contents in IoT domain are ephemeral and short-lived due to service and consumption characteristics. Moreover, they are usually freshly-generated with small size and different priorities. For instance, long and variable-length names in hierarchical schemes are not suitable in that case [4].

ICN MEC relies on intensive in-network caching. However, caching challenges in ICN MEC are manifold: The cache management for a large-scale distributed system as MEC environment is complex. Therefore, an effective ICN 
TABLE II: Challenges and research directions for ICN and MEC integration in 5G edge.

\begin{tabular}{|l|l|}
\hline Challenge & Research directions \\
\hline \hline Integration and deployment & Define common use cases, well-defined APIs \\
\hline Standardization & Common standards, collaborative objectives, collaborative research projects \\
\hline Context awareness & MEC can enable context-aware localized services. ICN can support MEC providing proactive caching. \\
\hline ICN MEC orchestration & AI techniques for autonomic network management, standardization, development of common APIs \\
\hline Support for different applications & $\begin{array}{l}\text { Mobile applications are designed in client-server architecture for a connection-oriented setting. This characteristic } \\
\text { has to transform to a more peer-driven setup. }\end{array}$ \\
\hline Network slicing for ICN segments & $\begin{array}{l}\text { For proliferation of ICN in the edge, we need ICN segments and network slicing. Agile management and scalability } \\
\text { are related challenges. }\end{array}$ \\
\hline $\begin{array}{l}\text { Control signaling for cache manage- } \\
\text { ment in information-centric edge }\end{array}$ & $\begin{array}{l}\text { Since caching infrastructure is a distributed system, the availability of information such as metric values can } \\
\text { render a caching management scheme infeasible when those data are not accessible. }\end{array}$ \\
\hline Security and privacy & $\begin{array}{l}\text { ML techniques for threat detection and situational awareness, MEC driven ICN traffic isolation techniques, AI } \\
\text { driven security management, user-level authorization and access control mechanisms }\end{array}$ \\
\hline Naming & Scalability, structure selection, redundancy minimization, versioning \\
\hline
\end{tabular}

caching scheme should exhibit computational efficiency in MEC. Moreover, the more data support needed for cache management, the lower feasibility and the higher overhead. Since caching infrastructure is a distributed system, such data requirements should be minimized [13]. The provisioning of proper storage capabilities to MEC nodes is also an resource allocation problem in heterogeneous networks.

Possible Solutions: One approach for dynamic object naming can be hash-based names with indirect binding. Versioning is one possible solution for updating named-data object since it can be replicated and stored in in-network storage for later retrieval but they need to be updated. Naming devices can be useful in an IoT system to access a particular device. A friendly way to address a device with a persistent name is to use a contextual hierarchical name, which is done a priori based on a predefined naming convention. Semantic and metadata-based content names are also possible. A semanticbased naming approach can allow for successful retrieval of name through a set of keywords. Moreover, enriching contents with metadata allows to better describe the names and to establish association between similar ones.

\section{E. Mobility}

Mobility support and session-less transport are inherent in ICN since contents rather than end-points are crucial to access and consume data generated by dense networks with massive connectivity. Although consumer mobility is supported by caching, producer mobility is yet to be addressed to allow seamless mobility in edge services. Application mobility for a stateful service relies on transfer and synchronization of the service state between the original and relocated application instance(s) to enable service continuity [11]. This is challenging since it is necessary to have multiple application instances running concurrently with seamless state transfer between source and other instances. Additionally, optimal link selection for wireless transport is a difficult problem in a cross-layer structure, i.e., content should be available to an queried anchor while the channel conditions should also be favorable. For heterogeneous edge networks with ICN and ICN-less modes, synchronization and handover of mobile nodes between these segments is another challenging research question.

Possible Solutions: In an ICN MEC converged network, the mobility can be offered as a service that is only activated when a particular user, content, or conditions require it. The formulation of an analytical model for ICN mobility support management will be another solution. In order to achieve this, it will be important to investigate the movement pattern of mobile content producers in the mobile network. Moreover, the mobility management can be tightly coupled with network management aspects with respect to policy enforcement, link control, and other parameters necessary for establishing the node's link to the network.

\section{STANDARDIZATION}

Globally, there has been a sizeable set of standardization bodies that are contributing immensely to define $5 \mathrm{G}$ technologies such as radio access, MEC, service management, and network architecture. Among them, ETSI is the main responsible standardization entity for MEC. In 2014, ETSI has launched the Industry Specification Group (ISG) to standardize MEC concept and specify its operation ${ }^{1}$. ETSI MEC ISG has been developing ETSI GS MEC 003 Framework and Reference Architecture for detailing MEC reference architecture with the latest V2.1.1 update in 2019. Similarly, ICN is mainly standardized by Internet Engineering Task Force (IETF). In 2012, IETF has started an new group called ICN Research Group (ICNRG) ${ }^{2}$ to couple ongoing ICN research with the solutions that are relevant for evolving the Internet at large. For mobile networks, IETF ICNRG has developed the Internet Draft Native Deployment of ICN in LTE, 4G Mobile Networks for ICN integration into 4G networks in 2019. These two organizations have a large number of common partners, both academic and industry who can push forward the ICN MEC integration for 5G. Moreover, ITU-T SG $13^{3}$ has been

\footnotetext{
${ }^{1}$ ETSI Multi-access Edge Computing (MEC) ISG: https://www.etsi.org/ technologies/multi-access-edge-computing

${ }^{2}$ IETF ICN Research Group (icnrg): https://datatracker.ietf.org/rg/icnrg/ documents/

${ }^{3}$ ITU-T Study Group 13: https://www.itu.int/en/ITU-T/about/groups/Pages/ sg13.aspx
} 
doing work on ICN standardization regarding requirements and architecture for future networks. This ITU standardization activity has also direct impact on $5 \mathrm{G}$ networks since it includes work for exploring how to integrate ICN paradigm into IMT-2020 networks [14]. Accordingly, ITU-T SG 13 has been actively working on the recommendation Y.ICNEdge: Architecture of ICN-enabled edge network in IMT-2020 which specifies the requirements and architecture about ICNenabled edge network in IMT-2020. The latest update to the recommendation was done in late 2019. There are also opensource initiatives such as Cisco's hICN named after the Open Source project created in the Linux Foundation Networking (LFN) under the Fast Data (FD.io) umbrella [15].

Conceptually, ICN falls under a different technology approach since it is essentially related to the Internet rather than service provider networks. Thus, ICN MEC integration requires cross-domain industry collaborations such as joint research projects and support from these standard developing organizations. The ITU-T SG 13 is an important first effort to consider ICN and MEC in a holistic way for standardization. In that regard, potential ETSI and IETF collaboration would be beneficial and boost impact since these would facilitate this synergy and increase efficiency. Moreover, ITU can contribute to a greater extent, especially from the ICN aspect, as the overarching standardization body.

\section{Future RESEARCH DiRECTIONS FOR BEyOND 5G (B5G) NETWORKS}

Edge computing trend including MEC is expected to grow and even become an indispensable part of in B5G networks. In B5G MEC applications, one of the key underlying characteristics is the inherent need for intelligence. Therefore, ML/AI techniques will play a key role in ICN MEC integrated B5G networks [16]. Future MEC based user applications in B5G networks will become increasingly reliant on AI (e.g. fully autonomous transportation). In addition, MEC based intelligence is necessary to automate the control and management functionalities (e.g., complex channel scheduling schemes, dynamic densification of cells) in RANs. Such deployments can lead to MEC based near-real time Radio Intelligent Controller (RIC) in B5G networks. To support such kind of intelligence, MEC deployments will need to rely on network architectures beyond IP. Here, ICN is an potential candidate with its obvious benefits such as fast and efficient data delivery and improved reliability.

Moreover, ML techniques and AI building on ICN and MEC seem to be promising tools to solve most of the discussed challenges regarding their integration in Section IV. The impact of these challenges will be exacerbated with massive connectivity and immense volume of generated data. In such environments, ICN networks need to realize context awareness, timely response and traffic offloading for B5G applications. To support that, MEC will require unified orchestration of the edge network resources such as computing, networking and caching. AI will be helpful to control and manage computing in the MEC environment whereas ML can be used to analyze high level statistics for context description, real-time observations, and user feedback, and support these control frameworks. ML approaches can assist the processing of content prefix in ICN by supporting efficient classification in the application and network levels. AI-based control and self-learning systems will be useful for handover, load balancing and routing decisions based on both the network's and the end user's QoS information.

The inclusion of new bandwidth-hungry network services will exponentially increase the data transfer in B5G networks. Handling of such big data is one of the interesting challenges in B5G. Both ICN and MEC offer features to tackle this challenge. For instance, MEC can eliminate the requirement of sending the raw data to the centralized cloud and ICN can efficiently distribute the data within the network infrastructure. Therefore, the utilization of ICN MEC for big data handling in B5G is a worthwhile research area.

At the infrastructure layer, network softwarization (SDN/NFV) provides capabilities for implementing ICN MEC due to more flexible forwarding and control schemes. A good example is the creation of a network slice including MEC for ICN operation. However, the role of these technologies and how they can facilitate ICN MEC efficiently (in addition to their utility for other purposes) are open research topics in future networks. At the radio access side, cross-layer designs where content delivery and caching are optimized for PHY/MAC layer conditions (e.g. use MEC nodes with favorable channel conditions for ICN caching) are promising future research directions. In that regard, novel transmission schemes such as NOMA can be used to improve ICN MEC offloading in joint optimization schemes. Similarly, collocation of MEC and C-RAN can enable MEC services to exploit C-RAN for optimizing ICN operation with additional context information in RAN. At the application layer of B5G networks, ICN MEC can enable fast and efficient content dissemination for various vertical scenarios. For instance, it is beneficial in mission-critical UAV networks to tackle challenges such as high mobility, heterogeneous communication protocols, security vulnerabilities, and strict delay constraints. However, the pros and cons of ICN MEC need to be further investigated to serve those verticals in an optimized way.

\section{CONCLUSION}

In this work, we first present and discuss ICN and MEC concepts regarding their envisaged roles in $5 \mathrm{G}$ realization. Both paradigms are posed to be integral elements of future Internet ecosystem. Nevertheless, how they are going to be utilized and implemented in 5G and Beyond networks are still open questions. We also elaborate on the implications of ICN and MEC integration and discuss emerging benefits as well as challenges and solutions. Finally, we present important standardization efforts and future research directions towards Beyond 5G networks with realization of ICN and MEC adoption.

\section{REFERENCES}

[1] A. V. Vasilakos, Z. Li, G. Simon, and W. You, "Information Centric Network: Research Challenges and Opportunities," Journal of Network and Computer Applications, vol. 52, pp. 1-10, 2015. 
[2] B. Wissingh, C. A. Wood, A. Afanasyev, L. Zhang, D. R. Oran, and C. Tschudin, "Information-Centric Networking (ICN): CCNx and NDN Terminology," Internet Engineering Task Force, Internet-Draft draft-irtficnrg-terminology-08, Jan. 2020, work in Progress. [Online]. Available: https://datatracker.ietf.org/doc/html/draft-irtf-icnrg-terminology-08

[3] R. Ravindran, A. Chakraborti, S. O. Amin, A. Azgin, and G. Wang, "5G-ICN: Delivering ICN Services over 5G Using Network Slicing," IEEE Commun. Mag., vol. 55, no. 5, pp. 101-107, 2017.

[4] S. Arshad, M. A. Azam, M. H. Rehmani, and J. Loo, "Recent advances in information-centric networking-based internet of things (icn-iot)," IEEE Internet of Things Journal, vol. 6, no. 2, pp. 2128-2158, April 2019.

[5] "Understanding Information-Centric Networking and Mobile Edge Computing," 5G Americas, December 2016, accessed on 12.01.2018. [Online]. Available: http://www.5gamericas.org/files/1214/8175/3330/Understanding Information_Centric_Networking_and_Mobile_Edge_Computing.pdf

[6] E. Ahmed, M. Imran, M. Guizani, A. Rayes, J. Lloret, G. Han, and W. Guibene, "Enabling mobile and wireless technologies for smart cities," IEEE Commun. Mag., vol. 55, no. 1, pp. 74-75, 2017.

[7] M. Maier, M. Chowdhury, B. P. Rimal, and D. P. Van, "The Tactile Internet: Vision, Recent Progress, and Open Challenges," IEEE Communications Magazine, vol. 54, no. 5, pp. 138-145, 2016.

[8] P. Porambage, J. Okwuibe, M. Liyanage, M. Ylianttila, and T. Taleb, "Survey on multi-access edge computing for Internet of Things realization," IEEE Commun. Surveys Tuts., vol. 20, no. 4, pp. 2961-2991, 2018.

[9] D. Grewe, M. Wagner, M. Arumaithurai, I. Psaras, and D. Kutscher, "Information-centric mobile edge computing for connected vehicle environments: Challenges and research directions," in Proceedings of the Workshop on Mobile Edge Communications. ACM, 2017, pp. 7-12.

[10] H. Jin, D. Xu, C. Zhao, and D. Liang, "Information-centric mobile caching network frameworks and caching optimization: a survey," EURASIP Journal on Wireless Communications and Networking, vol. 2017, no. 1, p. 33, 2017.

[11] S. Kekki, W. Featherstone, Y. Fang, P. Kuure, A. Li, A. Ranjan, D. Purkayastha, F. Jiangping, D. Frydman, G. Verin, K.-W. Wen, K. Kim, R. Arora, A. Odgers, L. M. Contreras, and S. Scarpina, "MEC in 5G networks," ETSI White Paper, 2018.

[12] R. Ravindran, P. Suthar, A. Chakraborti, S. O. Amin, A. Azgin, and G. Wang, "Deploying ICN in 3GPP's 5G nextgen core architecture," in 2018 IEEE $5 G$ World Forum (5GWF). IEEE, 2018, pp. 26-32.
[13] G. Gür, "Energy-aware cache management at the wireless network edge for information-centric operation," Journal of Network and Computer Applications, vol. 57, pp. 33 - 42, 2015.

[14] ITU-T SG-13, "Requirements and Mechanisms of Transport Layer for Information Centric Networking in IMT-2020," ITU-T SG-13 Recommendation (under study), 2020, accessed on 02.19.2020. [Online]. Available: https://www.itu.int/itu-t/workprog/wp item.aspx?isn=14915

[15] G. Carofiglio, L. Muscariello, J. Augé, M. Papalini, M. Sardara, and A. Compagno, "Enabling ICN in the internet protocol: Analysis and evaluation of the Hybrid-ICN architecture," in ACM SIGCOMM ICN 2019, 2019.

[16] B. Aazhangaaaa, P. Ahokangas, H. Alves, M.-S. Alouini, J. Beek, H. Benn, M. Bennis, J. Belfiore, E. Strinati, F. Chen et al., "Key Drivers and Research Challenges for 6G Ubiquitous Wireless Intelligence (White Paper)," Oulu, Finland: 6G Flagship, University of Oulu, vol. 1, 2019.

Gürkan Gür is a senior lecturer at Zurich University of Applied Sciences (ZHAW), Switzerland. He received his B.S. degree in electrical engineering in 2001 and Ph.D. degree in computer engineering in 2013 from Bogazici University. His research interests include Future Internet, information security, next-generation wireless networks and ICN.

Pawani Porambage Pawani Porambage is a postdoctoral researcher at the Centre for Wireless Communications, University of Oulu, Finland. Her main research interests are lightweight security protocols, security and privacy on IoT \& MEC, and WSNs.

Madhusanka Liyanage is an Ad Astra Fellow/ Assistant Professor at School of Computer Science, University College Dublin, Ireland. He is also an adjunct professor at the University of Oulu, Finland. His research interests are SDN, IoT, Blockchains, MEC, mobile and network security. More info: http://www. madhusanka.com 